\title{
Numerical and Analytical Assessment of a Coupled Rotating Detonation Engine and Turbine Experiment
}

\author{
Daniel E. Paxson* \\ NASA Glenn Research Center, Cleveland, Ohio, 44130 \\ Andrew Naples ${ }^{\dagger}$ \\ Innovative Scientific Solutions Inc. Dayton, OH, 45440
}

\begin{abstract}
An analysis is presented of an experimental rig comprising a rotating detonation engine (RDE) with bypass ejector flow coupled to a downstream turbine. The analysis used a validated computational fluid dynamics RDE simulation combined with straightforward algebraic mixing equations for the bypass flow. The objectives of the analysis were to supplement and interpret the necessarily sparse measurements from the rig, and to assess the performance of the RDE itself, which was not instrumented in this installation. The analysis is seen to agree reasonably well with available data. It shows that the RDE is operating in an unusual fashion, with subsonic flow throughout the exhaust plane. The detonation event itself is producing a total pressure rise relative to the pre-detonative pressure; however, the length of the device and the substantial flow restriction at the inlet yield an overall pressure loss. This is not surprising since the objective of the rig test was primarily aimed at investigating RDE/turbine interactions, and not on performance optimization. Furthermore, the RDE was designed for fundamental detonation studies and not performance. Nevertheless, the analysis indicates that with some small alterations to the design, an RDE with an overall pressure rise is possible.
\end{abstract}

\section{Nomenclature}

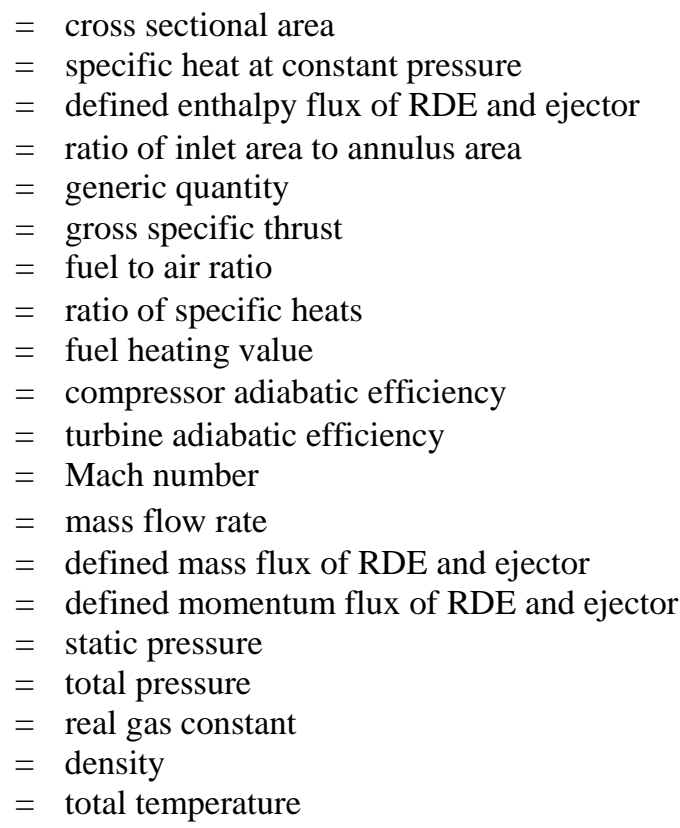

*Aerospace Research Engineer, Research and Engineering Directorate, 21000 Brookpark Road/MS 77-1, AIAA Associate Fellow

${ }^{\dagger}$ Research Engineer, 7610 McEwen Rd, AIAA Member 


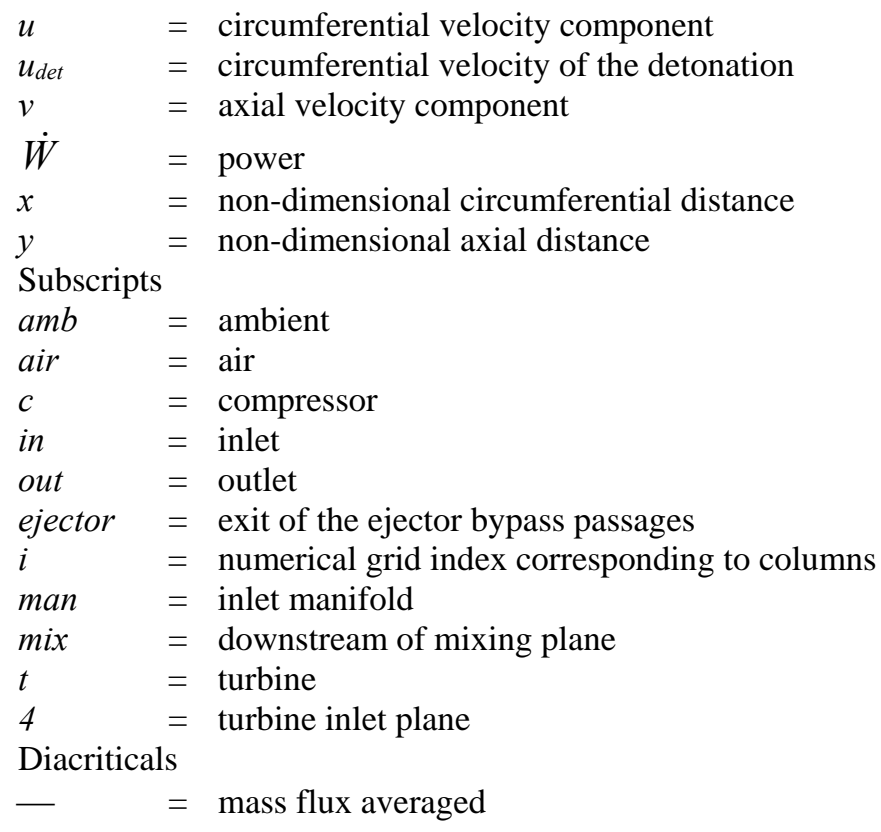

\section{Introduction}

$\mathrm{T}$ The rotating detonation engine (RDE) is currently under investigation as an approach to achieving pressure gain combustion (PGC) for propulsion and power systems. The RDE essentially consists of an annulus with one end open (or having a nozzle) and the other end valved (typically using non-mechanical, fluidic means to promote throughflow and prevent backflow). Fuel and oxidizer enter axially through the valved end. The detonation travels circumferentially. Combustion products exit predominantly axially through the open end. The majority of the fluid entering the device is passed over by the rotating detonation wave which, as a form of confined heat release, substantially raises the pressure and temperature. The fluid is then expanded and accelerated as it travels down the annulus. Ideally, the flow exiting the device has a higher average total pressure than the flow that enters; though the averaging must be done with care due to the non-uniformity of the flow.

The pressure gain of an RDE can be utilized by a nozzle and produce thrust directly, or it can be utilized by a turbine to produce work. The latter application can, in theory, produce additional work when compared to that of a conventional combustor which incurs a pressure loss when operating at the same inlet conditions and fuel/air ratio. However, there is a caveat to the RDE/turbine application. The RDE exit flow is highly non-uniform, both spatially and temporally. The impact of this characteristic on turbine performance is not known, but is generally assumed to be detrimental, at least for turbines which are designed for uniform flows ${ }^{1}$.

In order to investigate coupled RDE and turbine operability, an experimental rig was fabricated and run at the Air Force Research Laboratory (AFRL) facility in Dayton, Ohio. The rig consisted of a modified Allison T63 gas turbine engine shown unmodified in Fig. $1^{2-5}$. The normal liquid-fueled combustor was replaced by a hydrogen-fueled RDE and ejector combination. The RDE and ejector were designed for the rated gas turbine flow rate and nominal turbine inlet temperature.

Although these tests were focused on turbine operability rather than system performance, the question subsequently arose as to whether the RDE and ejector combination could be modeled and the measured quantities matched. Such a model would provide insights into the physics of the combustion system. Furthermore, it could supplement the limited instrumentation available, and perhaps help develop a next generation test that yielded improved performance (even if it was not the primary focus of the experimental effort). This paper describes a preliminary attempt at such a model and presents the results and analysis therefrom.

Additional and necessary details of the experimental setup will first be presented. The model, which combines the output of an existing computational fluid dynamic RDE code with algebraic mixing calculations appropriate to ejectors, will then be described. Comparison with experimental results will then be presented.

The model output will then be examined in order to illustrate the unique manner in which the RDE was operating, and to highlight the major sources of lost availability (i.e. entropy production). The paper concludes with some 


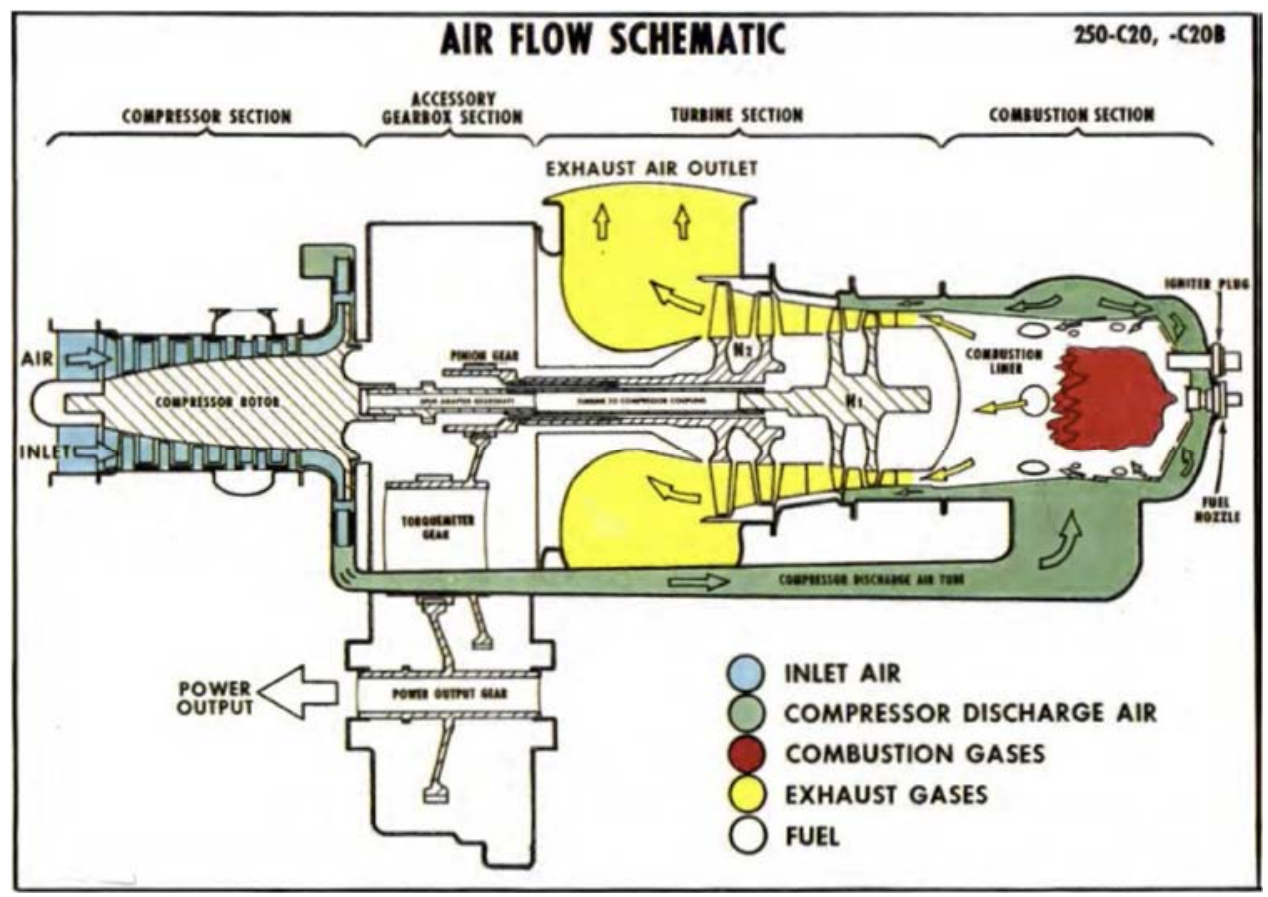

Figure 1. Allison T63 schematic from Ref. 2

suggested modifications to the RDE component in order to mitigate loss, and quantifies the performance enhancement these modifications might provide.

\section{Experimental Description}

A detailed description of the experiment and results may be found in Ref. 5. The focus in the paper is on the analysis. As such the rig description will contain only the elements essential to that effort.

The RDE and ejector components are shown schematically in Fig. 2. The RDE and ejector were fed from a pressurized facility tank with enough air for approximately 15 minutes of operation. The T-63 compressor outflow was decoupled from the engine and vented from the facility through a controllable valve. This decoupled (aka openloop) arrangement allowed the compressor to function as a measurable load for the gas generator turbine (i.e. a kind of dynamometer), which is on the same shaft as the compressor. The compressor was fed by ambient air. The downstream power turbine, which is on a separate shaft, was coupled to an actual dynamometer to measure its power. During testing the compressor discharge valve was adjusted such that the mass flow rate through the compressor matched the mass flow rate through the RDE and ejector combination. However, the compressor discharge pressure and temperature did not match the values used in the RDE and ejector inlet manifolds. This mode of testing anticipates comparison with future closed loop testing where, through improvements in RDE design, the required inlet manifold conditions might more closely match those of the compressor discharge.

The complete test setup is shown in Fig. 3. It is evident that the combination of utilizing a very compact commercial gas turbine, operating open-loop, installing a dynamometer, and the manner by which the RDE and ejector were mated made instrumentation exceedingly difficult. The difficulties were compounded by the fact that the RDE materials were operating near their limits and any penetrations for instrumentation may have exceeded them. As a result, the only measured quantities for any of the operating points examined were time-averaged inlet air and fuel mass flow rates, inlet manifold pressures and temperatures, shaft speeds, compressor discharge pressure and temperature, power turbine power, and a single static pressure just upstream of the turbine inlet (see Fig. 2). This made subsequent numerical and analytical assessment a challenge, and the results necessarily speculative. Nevertheless, the effort did provide insight into RDE operations in the gas turbine environment and is therefore of value to report.

\section{Model Description}

The RDE component is simulated using the computational fluid dynamic (CFD) tool described briefly in the following sub-section. Output from the CFD tool is combined with the bypass flow (see Fig. 2) using constant area 
mixing calculations to yield a single mixed fluid state and Mach number for the ejector. This is then accelerated through the contraction to yield the turbine inlet conditions.

\section{A. RDE Simulation}

The RDE simulation used in this paper has been detailed in the literature ${ }^{6-9}$, and will only be briefly described here. The basis is a high resolution, computational fluid dynamic (CFD) algorithm that integrates the quasi-two-dimensional, single-species, reactive Euler equations with source terms. The code adopts the detonation frame of reference and deliberately utilizes a coarse grid (i.e. is diffusive) in order to eliminate the highest frequency unsteadiness (e.g. detonation cells, Kelvin-Helmholtz phenomena). The result is a flowfield solution that is invariant with time when converged. The working fluid is assumed to be a single, calorically perfect, premixed gas. The detonation speed is imposed in this formulation. The appropriate value to impose, as found through iteration, is that which yields the time-invariant solution.

The source terms contain sub-models which govern the reaction rate, momentum losses due to skin-friction, and the effects of heat transfer to the walls. The sub-models are adapted from validated one-dimensional sub-models used to investigate pulse detonation engines and other gasdynamic devices ${ }^{10-12}$.

The governing equations are integrated numerically in time using an explicit, second-order, two-step, Runge-Kutta technique. Spatial flux derivatives are approximated as flux differences, with the fluxes at the discrete cell faces evaluated using Roe's approximate Riemann solver. Second-order spatial accuracy (away from discontinuities) is obtained using piecewise linear representation of the primitive variable states within the cells (aka Monotonic UpstreamCentered Scheme for Conservation Laws, or MUSCL). Oscillatory behavior is avoided by limiting the linear slopes.

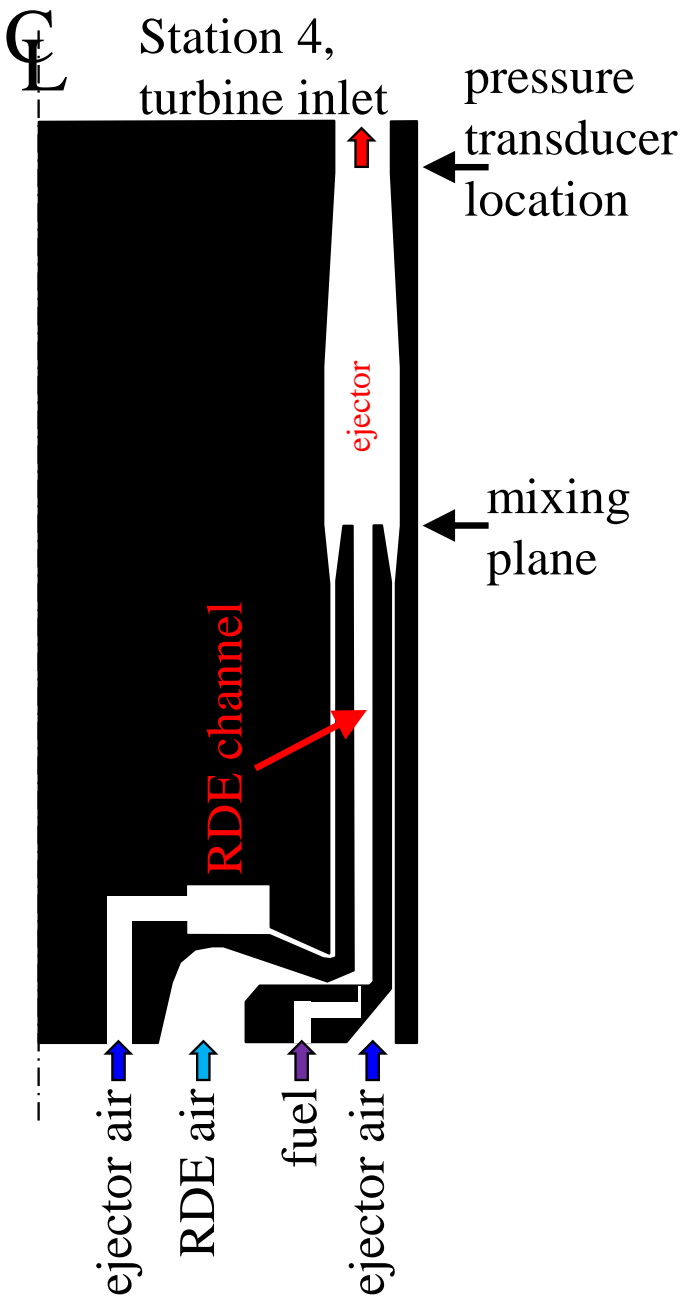

Figure 2. RDE and ejector schematic

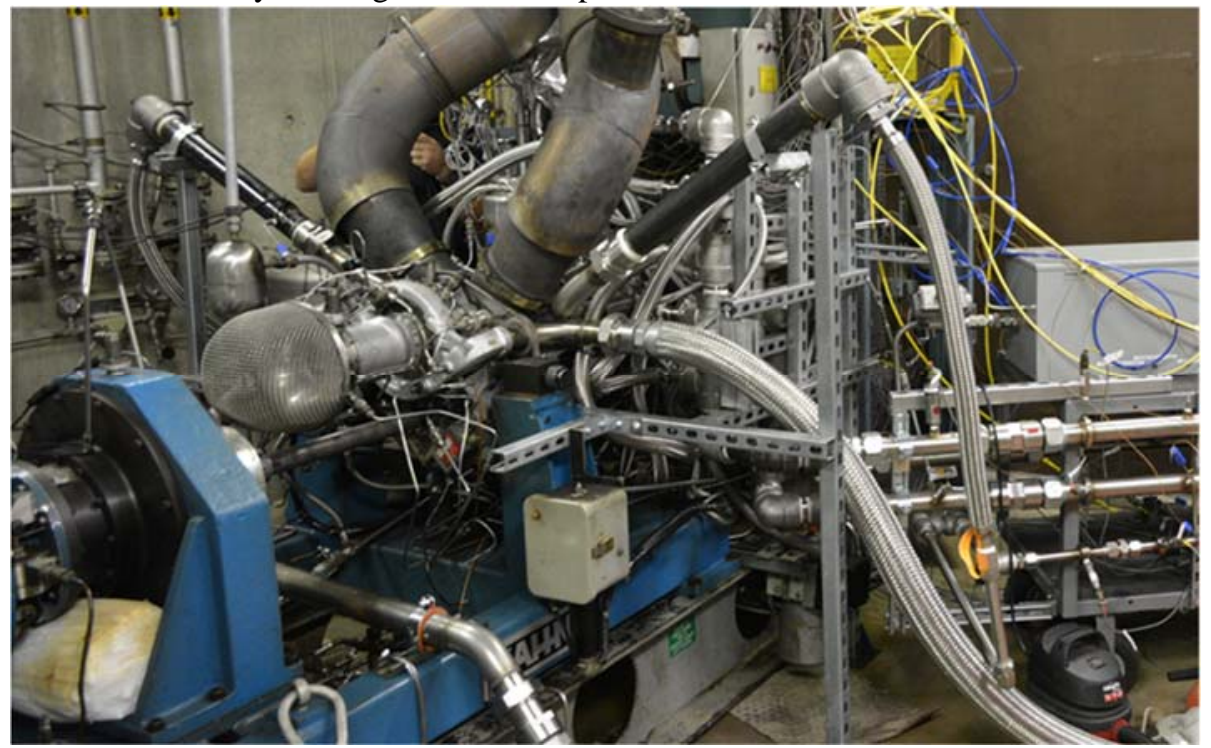

Figure 3. Complete experimental setup

4

American Institute of Aeronautics and Astronautics 
Considering an 'unwrapped' RDE annulus where the non-dimensional circumferential direction is $x$, and the axial direction is $y$, the following boundary conditions are imposed. At $x=0.0$ and $x=1.0$, periodic (aka symmetric) conditions are used. These insure that the $\mathrm{x}$-dimension of the computational space faithfully represents an annulus (which is continuous and has no boundary). At $y=y_{\max }$, constant pressure outflow is imposed along with characteristic equations to obtain $\rho$ and $v$ for the image cells. If the resulting flow is sonic or supersonic, then the imposed pressure is disregarded. In addition, if the upstream flow is supersonic, then $p, \rho$, and $v$ are extrapolated from the interior ${ }^{13,14}$. The possibility for a normal shock solution whereby supersonic outflow jumps to subsonic is also accommodated. In some extreme subsonic scenarios, inflow is possible in this presumed outflow plane. The boundary condition logic can accommodate this scenario as well. The x-velocity component $u$ is extrapolated from the interior at each boundary location. At $y=0.0$ (the inflow face), partially open boundary conditions are applied as described and validated in Ref. 14. This face is presumably fed by a large manifold at a fixed total pressure and temperature. The manifold terminates at the face and is separated from it via an orifice. The ratio of orifice flow area to RDE annulus area, $\varepsilon$ is generally less than 1 . If the interior pressure is less than the manifold pressure, $p_{\text {man }}$, then inflow occurs. The boundary condition routine determines $p, \rho$, and $v$ for the inflow face image cells subject to a momentum (total pressure) loss model which depends on the mass flow rate and the value of $\varepsilon$. It is capable of accommodating a scenario where the orifice becomes choked. The $\mathrm{x}$-velocity component $u$ is prescribed during inflow, and it is here that a reference frame change is implemented. Rather than specify $u=0$ (i.e. no swirl) which is the laboratory or fixed frame condition, the negative of the detonation speed, $u_{\text {det }}$ is prescribed instead. As a result of this change to the detonation reference frame, the computational space becomes one where a steady-state solution is possible. If the interior pressure along the inlet face is greater than $p_{\operatorname{man}}$, as might be found just behind the detonation, then there will be backflow into the manifold through the orifice. The boundary condition routine can accommodate this as well.

In RDE simulations where inlet backflow occurs, the total mass and enthalpy that flow backward are averaged over the circumferential backflow span (recalling that in the steady detonation frame of reference, time is simply span divided by detonation velocity). When the interior pressure subsequently drops below $p_{\operatorname{man}}$ and forward flow resumes, all of the mass that flowed backward is sent back into the RDE at the same average enthalpy that it exited. Once this mass has re-entered, the prescribed manifold premixed air and fuel mixture enthalpy is used.

Although the model assumes that premixed air and fuel enter through the inlet, the reality of most RDE experiments is that fuel and air are injected separately. This creates the possibility that some finite time (and associated convection distance) is required to mix before reaction can occur. As a crude model of this observed effect, the simulation provides a user specified number of axial computational cell rows near the inlet that do not react, even though the threshold temperature is reached. The delay can impact the amount of backflow to the inlet manifold, and the overall wave structure within the annulus. These, in turn, can impact performance. For the present work, this delay region was specified as being between $2 \%$ and $6 \%$ of the axial length. This range was chosen based on comparisons of the simulation output with highly instrumented RDE rigs ${ }^{7-9}$.

A converged solution of the RDE channel contains the states and velocity components of every numerical cell. Of particular interest for this analysis is the exit plane since it provides input for the mixing calculations to be described next. Among many useful quantities that may be computed in this plane is the gross specific thrust. Based on the uniform grid spacing used in the simulation the calculation is written as follows.

$$
F_{s p g}=\frac{\sum_{1, N}\left(p_{i}-p_{e x i t}+\rho_{i} v_{i}^{2}\right)}{\sum_{1, N} \rho_{i} v_{i}}
$$

In this equation, $p_{\text {exit }}$ is the imposed static pressure representing the exit boundary condition of the RDE. It is assumed to be a constant value spanning the entire mixing plane region.

\section{B. Mixing Calculation}

Considering the mixing plane shown in Fig. 2, and assuming a perfect gas for each flow, the following equations conserving mass, momentum, and energy flux can be written.

$$
\rho_{m i x} v_{m i x}=\frac{\left(\dot{m}_{R D E}+\dot{m}_{\text {ejector }}\right)}{A_{m i x}} \equiv m f
$$




$$
\begin{gathered}
p_{\text {mix }}+m f v_{\text {mix }}=\frac{\left(p_{\text {exit }} A_{\text {mix }}+\dot{m}_{R D E} F_{\text {spg }}+\gamma_{\text {air }} M_{\text {ejector }}^{2} p_{\text {exit }} A_{\text {ejector }}\right)}{A_{\text {mix }}} \equiv m o m \\
\frac{\gamma_{\text {mix }}}{\gamma_{\text {mix }}-1} p_{\text {mix }} v_{\text {mix }}+m f v_{\text {mix }}^{2}=\frac{\left(c_{p_{-} \text {air }} \dot{m}_{\text {ejector }} T_{t_{-} \text {ejector }}+c_{p_{-} R D E} \dot{m}_{R D E} \bar{T}_{t_{-} R D E}\right)}{A_{\text {mix }}} \equiv e f
\end{gathered}
$$

Equations 2-4 can be rearranged into a quadratic equation in $v_{\text {mix }}$.

$$
v_{m i x}^{2} m f \frac{\gamma_{m i x}+1}{2\left(\gamma_{m i x}-1\right)}-v_{m i x} m o m \frac{\gamma_{m i x}}{\left(\gamma_{m i x}-1\right)}+e f=0
$$

The subsonic root of Eq. 5 is the correct solution since it yields an increase in entropy compared to the mass flux average entropy of the separate streams. Substitution back into Eqs. 2 and 3 yields the remainder of the mixed information. The mixed temperature is found from the equation of state.

Several of the variables used in Eqs. 2-4 were not measureable directly from the rig, or calculated by the simulation, and therefore had to be estimated. In particular, the total temperature and Mach number at the ejector exit, $T_{\text {t_ejector, }}$, and $M_{\text {ejector }}$ were found as follows. Since there are no temperature measurements available beyond the inlet manifolds, the mixed total temperature entering the turbine, $T_{t_{-} \text {mix }}$ was first estimated using the equilibrium code CEA ${ }^{15}$, the measured fuel-to-air ratio (using air flow through both the RDE and ejector), $f / a$, and the inlet manifold temperature, $T_{t_{-} \text {man }}$. From this temperature, an average mixture specific heat can be obtained with a simple energy balance.

$$
c_{p_{-} \text {mix }}=\frac{c_{p_{-} \text {air }} T_{t_{-} \text {man }}+h_{f} f / a}{T_{t_{-} \text {mix }}}
$$

With the mixture specific heat in hand, the ejector exit total temperature is found with another energy balance.

$$
T_{t_{-} \text {ejector }}=\frac{\left(c_{p_{-} \text {mix }}\left(\dot{m}_{\text {ejector }}+\dot{m}_{R D E}\right) T_{t_{-} m i x}-c_{p_{-} R D E} \dot{m}_{R D E} \bar{T}_{t_{-} R D E}\right)}{\dot{m}_{\text {ejector }} c_{p_{-} \text {air }}}
$$

In this equation (and Eq. 4) $\bar{T}_{t_{-} R D E}$ is the mass flux averaged total temperature at the exit plane of the RDE as calculated from the simulation, and $c_{P \_} R E E$ is the user specified constant value of specific heat used for RDE flowfield*. The mass flux average of any quantity in this paper is defined as:

$$
\bar{f}=\frac{\sum_{1, N}\left(f_{i} \rho_{i} v_{i}\right)}{\sum_{1, N} \rho_{i} v_{i}}
$$

The value for $M_{\text {ejector }}$ is found from standard compressible flow relations as follows.

* The value for $\gamma_{\text {mix }}$ in Eq. 4 is found from the relationship $\gamma_{m i x}=\frac{c_{p_{-} m i x}}{\left(c_{p_{-} m i x}-R_{g_{-} m i x}\right)}$ where $R_{g_{-} \text {mix }}$ is the estimated mixture gas constant. 


$$
M_{\text {ejector }}=\sqrt{\frac{\sqrt{1+2 c_{p_{-} \text {air }} T_{t_{-} \text {ejector }}\left(\frac{\left(\gamma_{\text {air }}-1\right) \dot{m}_{\text {ejector }}}{\gamma_{\text {air }} A_{\text {ejector }} p_{\text {exit }}}\right)^{2}}-1}{\gamma_{\text {air }}-1}}
$$

The use of mass flux averaged temperature in Eq. 4 and gross specific thrust in Eq. 3 may, at first glance, give the appearance of a mixing calculation based on flows that have already been averaged. This would be inappropriate as it would 'pre-smooth' the flows to be mixed and necessarily lead to lower losses. However a close examination of Eqs. 1 and 8 show that, when multiplied by a mass flow rate as they are, both the mass flux averaged temperature and gross specific thrust deliver integrated quantities containing the full effects of non-uniformity to the mixing equations.

\section{Closure}

Both the mixing calculation and the RDE simulation require a key unknown for closure, and that is the exit plane static pressure, $p_{\text {exit }}$. Meanwhile, the only available pressure measurement was the static pressure at the turbine entrance, $p_{4}$ (see Fig. 2). As such, the following procedure is used. The measured upstream inlet manifold pressure and temperature are imposed as boundary conditions on the RDE simulation, and the average measured fuel/air mixture is prescribed. A guess is then made for the assumed constant $p_{\text {exit }}$. The effective RDE inlet throat area in the simulation is adjusted until the computed mass flow rate is within $3 \%$ of the measured value. The imposed $p_{\text {exit }}$ is also used in the mixing calculation, along with the output from the RDE calculation to calculate a mixed gas state and velocity. Assuming isentropic flow between the mixing plane and the turbine inlet plane where the pressure transducer is located, the Mach number at the turbine inlet may be found using the transcendental area Mach number equation.

$$
\frac{M_{4} A_{4}}{\left(1+\frac{\gamma_{\text {mix }}-1}{2} M_{4}^{2}\right)^{\frac{\gamma_{\text {mix }}+1}{2\left(\gamma_{\text {mix }}-1\right)}}}=\frac{M_{\text {mix }} A_{\text {mix }}}{\left(1+\frac{\gamma_{\text {mix }}-1}{2} M_{m i x}^{2}\right)^{\frac{\gamma_{\text {mix }}+1}{2\left(\gamma_{\text {mix }}-1\right)}}}
$$

Standard isentropic relationships may then be used to calculate the static pressure, $p_{4}$. The value obtained is then compared to the measured time-averaged static pressure of the transducer. The difference is used to make a new estimate for $p_{\text {exit. }}$. The closure process is repeated until the measured and calculated $p_{4}$ values match.

\section{Validation}

With so little instrumentation on the experiment, it is impossible to validate the modeling approach in any meaningful way. However, as a sort of 'reasonableness' check the model was applied to two of the operating points with parameters listed in Table 1. The relevant parameters for the mixing calculations and RDE simulation are shown in Table 2. The effective RDE inlet area required to achieve closure was then compared to actual area. For the $90 \%$ speed point, ratio of effective-to-actual area was 0.74 . For the $80 \%$ speed case it was 0.68 . Given the simplicity of the RDE inlet model, and the circuitous flow path of the actual inlet (which includes an abrupt transition from radial to axial flow), these ratios can be interpreted as discharge coefficients, and are quite reasonable when compared to other fluidic restrictions in the literature ${ }^{16}$.

In addition to this, limited data is available from Ref. 4. In this experiment, the exact same RDE was operated uninstalled in the engine, and with a somewhat different ejector arrangement. The RDE and ejector flow rates were very similar to the $90 \%$ speed point of Table 1 . The Ref. 4 experiment did not have a turbine inlet static pressure measurement on which to exercise the mixing calculation and determined a value of $p_{\text {exit }}$ to use in the simulation. As such, $p_{\text {exit }}$ was chosen such that its value, in ratio to the inlet manifold, was the same as the $90 \%$ speed point of Table 1. The ratio of effective-to-actual inlet area required to match the simulated and experimental RDE mass flow rates was found to be 0.79 ; very close to the $90 \%$ speed value of 0.74 reported above.

The Ref. 4 experiment had two time averaging pressure transducers (aka Capillary Tube Averaged Pressure, or CTAP's ${ }^{17}$ ) located $31 \%$ of the way axially down the RDE channel. They were arranged 180 degrees apart circumferentially in order to assess variations in averages in this direction. 
Table 1 Measured Parameters and Values at Two Operating Points

\begin{tabular}{|l|l|l|}
\hline Approximate \% Design Speed & 90 & 80 \\
\hline Ejector Air Flow Rate (lbm/s) & 1.81 & 1.63 \\
\hline RDE Air Flow Rate (lbm/s) & 0.66 & 0.46 \\
\hline Compressor Air Flow Rate (lbm/s) & 2.68 & 2.27 \\
\hline RDE Equivalence Ratio & 0.98 & 0.98 \\
\hline Overall Equivalence Ratio & 0.24 & 0.20 \\
\hline RDE Inlet Manifold Air Pressure (psia) & 86.2 & 67.6 \\
\hline Power Turbine Power (hp) & 168 & 86 \\
\hline Supply Air Temperature (R) & 460 & 460 \\
\hline Compressor Inlet Pressure (psia) & 14.7 & 14.7 \\
\hline Compressor Inlet Temperature (R) & 527 & 527 \\
\hline Compressor Discharge Pressure (psia) & 57.3 & 46.2 \\
\hline Compressor Discharge Temperature (R) & 877 & 811 \\
\hline Turbine Inlet Average Static Pressure (psia) & 64.9 & 52.8 \\
\hline Computed RDE exit plane pressure (psia) & 63.1 & 51.3 \\
\hline Calculated Turbine Inlet Temperature (R) & 1790 & 1562 \\
\hline Calculated Turbine Inlet Pressure (psia) & 67.0 & 54.4 \\
\hline
\end{tabular}

progressively larger at the lower gas-turbine speed points tested. Since the simulation is currently only designed to accommodate small levels of backflow, the lower speed test points were not examined. Details of only one test point are presented simply because the other simulation reveals no additional information.

The results to follow pertain to the $90 \%$ speed point described by the Table 1 measured parameter values. The compressor power (and therefore the gas generator turbine power) was calculated from the equation

$$
\dot{W}_{c}=\dot{m}_{c} c_{p_{-} \text {air }}\left(T_{t_{-} c_{-} \text {out }}-T_{t_{-} c_{-} \text {in }}\right)
$$

The value obtained was $319 \mathrm{hp.} \mathrm{As} \mathrm{a} \mathrm{check} \mathrm{on} \mathrm{the} \mathrm{calculation,} \mathrm{the} \mathrm{compressor} \mathrm{adiabatic} \mathrm{efficiency} \mathrm{was} \mathrm{calculated}$ from

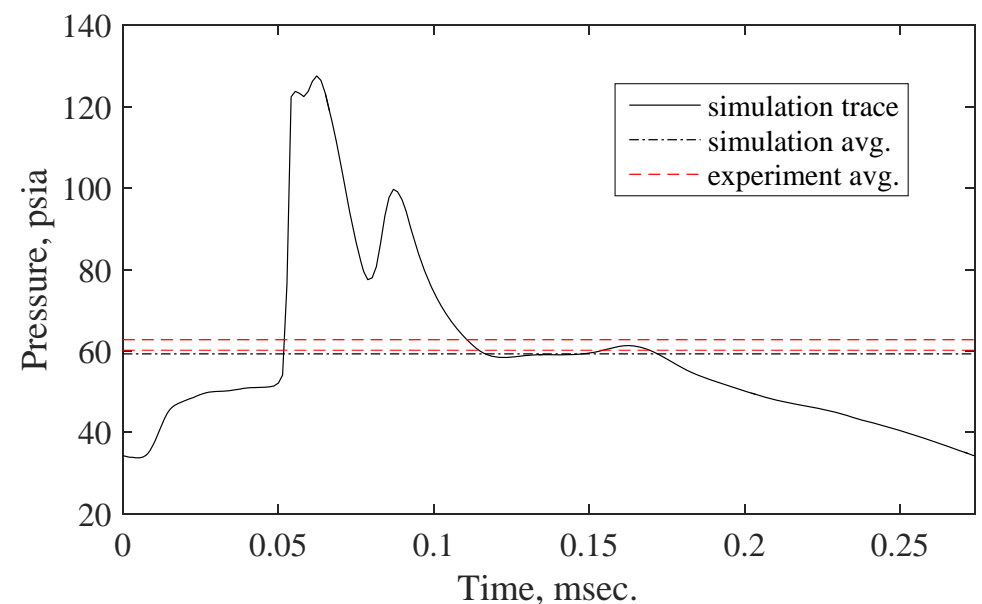

Figure 4. Simulated and two measured static channel pressures from the Ref. 4 experiment (180 deg. circumferential separation) at $31 \%$ of the RDE axial length.
Figure 4 shows the simulated pressure trace at this location (converted back from the detonation to the laboratory frame of reference). Also shown are the time average of this trace, and the experimental averages using the CTAP's. The agreement is quite good, particularly when considered in light of massive range of pressures indicated by the trace shown in Fig. 4.

\section{Results}

Four operating points were tested, and two were simulated; however, the details of only one simulated test point will be presented here. Only two were simulated because both showed evidence of backflow in the RDE exit plane which got
Table 2 RDE and Mixing Model Parameters

\begin{tabular}{|l|l|}
\hline$C_{p \_R D E}, \mathrm{ft}-\mathrm{lb}_{\mathrm{f}} / \mathrm{lb}_{\mathrm{m}} / \mathrm{R}$ & 354 \\
\hline$\gamma_{\mathrm{RDE}}$ & 1.264 \\
\hline $\mathrm{C}_{\mathrm{p} \_ \text {mix }}, \mathrm{ft}-\mathrm{lb}_{\mathrm{f}} / \mathrm{lb}_{\mathrm{m}} / \mathrm{R}$ & 205 \\
\hline$\gamma_{\operatorname{mix}}$ & 1.4 \\
\hline $\mathrm{C}_{\mathrm{p} \_ \text {air }}, \mathrm{ft}-\mathrm{lb} \mathrm{b}_{\mathrm{f}} / \mathrm{lb}_{\mathrm{m}} / \mathrm{R}$ & 187 \\
\hline Fuel heating value, BTU/lb $\mathrm{m}$ & 51,571 \\
\hline
\end{tabular}


total mass flow rate (see Table 1) were used to estimate the overall adiabatic turbine efficiency via the following equation.

$$
\eta_{t}=\frac{\dot{W}_{t}}{\left(\dot{m}_{\text {ejector }}+\dot{m}_{R D E}\right) c_{p_{-} m i x} T_{t_{-} m i x}\left(1-\left[\frac{p_{a m b}}{p_{t_{-} m i x}}\right]^{\frac{\gamma_{\text {mix }}-1}{\gamma_{\text {mix }}}}\right)}
$$

The calculated value was $\eta_{t}=0.83$. Again, no turbine efficiency data was available from the manufacturer for comparison. However, as with the compressor, the calculated value was consistent with NPSS maps, which indicate efficiency values between 0.86-0.90. This is remarkably good agreement considering the simplifications and assumptions involved in the analysis. More importantly, the efficiency is quite high given the apparent unsteadiness of the flow entering the turbine. Figure 5 shows a trace from the static pressure probe at the turbine inlet. The oscillations, presumably caused by the upstream RDE, show a peak-to-peak variation that is $22 \%$ of the mean value. Details of this measurement using the so-called 'ITP' transducer installation are found in Ref. 4.

One possible explanation for this apparent high turbine efficiency is that the lost work extraction capability normally associated with unsteadiness has already been accounted for through the entropy generation inherent in the mixing calculation. Nearly any type of mathematical smoothing or averaging of a non-uniform flowfield adds entropy ${ }^{1}$ and reduces work potential. The momentum preserving type used here appears to introduce the correct amount. In other words, the work of a relatively high efficiency turbine encountering a uniform, but high entropy flow, is roughly the same as the work done by lower efficiency turbine encountering a non-uniform, but lower entropy flow. It is important to note that this is likely a fortuitous result based on this particular flowfield and turbine. The flow from a pulse detonation engine, for example, sent into a high performance turbine may not yield such an equivalence. On the other hand, it is a positive sign for turbine coupled PGC technology that significant unsteadiness does not appear to severely compromise turbine performance.

\section{A. RDE Operation}

Examining the RDE component alone, the simulation output provides a number of interesting details of the flowfield. Figure 6 shows computed steady contours of temperature throughout the annulus so that the wave pattern and relative height of the detonation may be seen. The axial direction is represented by the variable $y$, and the circumferential direction by $x$. Only half of the circumference has been shown since there were two detonation waves present at this operating point. The variables $x$ and $y$ have each been normalized by one half of the circumference. The temperature has been normalized by the reference value of $520 \mathrm{R}$. Also shown in Fig. 5 is a streamline that traces the shortest path of a particle entering the computational space from the inlet to the exit. It is clear from this figure that the detonation height is low, and that axial fluid velocity is relatively low (i.e. a given fluid particle is resident for at least three passes of the detonation). This is a markedly different pattern than is normally seen in RDE simulations and illustrates the effect of having a relatively high exit back pressure compared to the inlet manifold pressure.

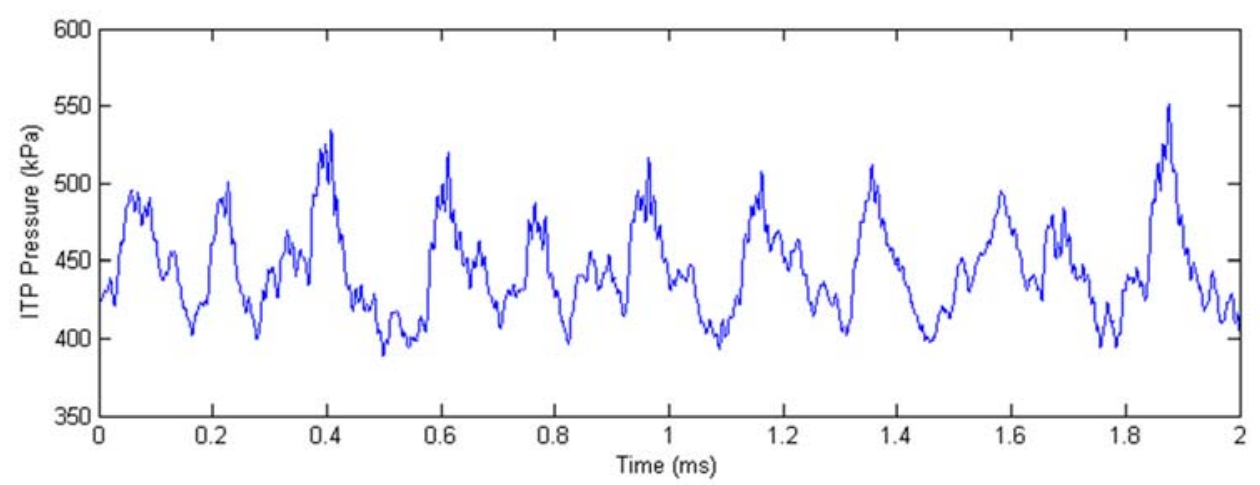

Figure 5. Static pressure trace at turbine inlet

American Institute of Aeronautics and Astronautics 


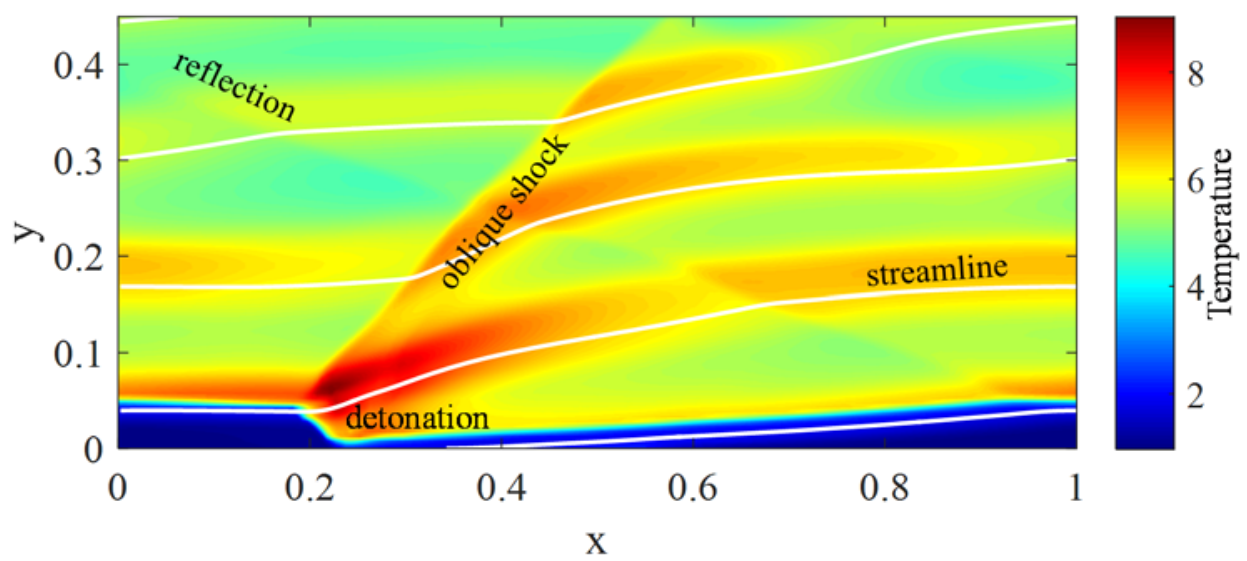

Figure 6. Computed contours of normalized temperature throughout the annulus of the experimental RDE, in the detonation frame of reference.

Figure 7 shows the exit plane axial Mach number and temperature of the RDE. Again, a highly unusual pattern is observed in that it is entirely subsonic, and there is actually a small amount of backflow observed. This too appears to be caused by the high back pressure.

The RDE used in the experiment is a laboratory scale, research unit, constructed to explore fundamental aspects of operation. As such it was not optimized for, nor expected to yield, high performance. It is no surprise then that the computed pressure ratio across the device, based on the so-called specific thrust equivalent exhaust total pressure ${ }^{1,19}$ was found to be 0.83 . Nevertheless it is worthwhile to examine the causes of the low performance, quantify their effect, and examine if simple changes might yield improved performance.

For example, it is observed from Fig. 6 that the RDE is significantly longer than necessary. The oblique shock which passes multiple times over the reacted gas does no useful work. It simply generates entropy. Additionally, the long gas path leads to significant losses through wall friction, and heat transfer. The simulation predicts that approximately $28 \%$ of the available chemical energy from fuel is lost to the walls. Figure 8 shows the mass fluxaveraged total pressure normalized by the inlet manifold pressure as a function of axial distance down the RDE channel. The lost availability is evident, as is the argument for shortening the RDE.

Turning to the inlet end of the RDE, it is noted that designs such as the one used in the experiment represent a trade-off between (among other factors) minimizing total pressure loss associated with forward flow through a restriction (see Fig. 2), and preventing backflow of high pressure gases that exist immediately behind the rotating detonation wave. The computed normalized mass flux at the inlet of the RDE is shown in Fig. 9. The evident backflow is approximately $18 \%$ of throughflow. Losses due to this backflow are difficult to quantify; however, it is intuitive that momentum generated in the upstream direction is not beneficial. Furthermore, it is clear that any backflow must

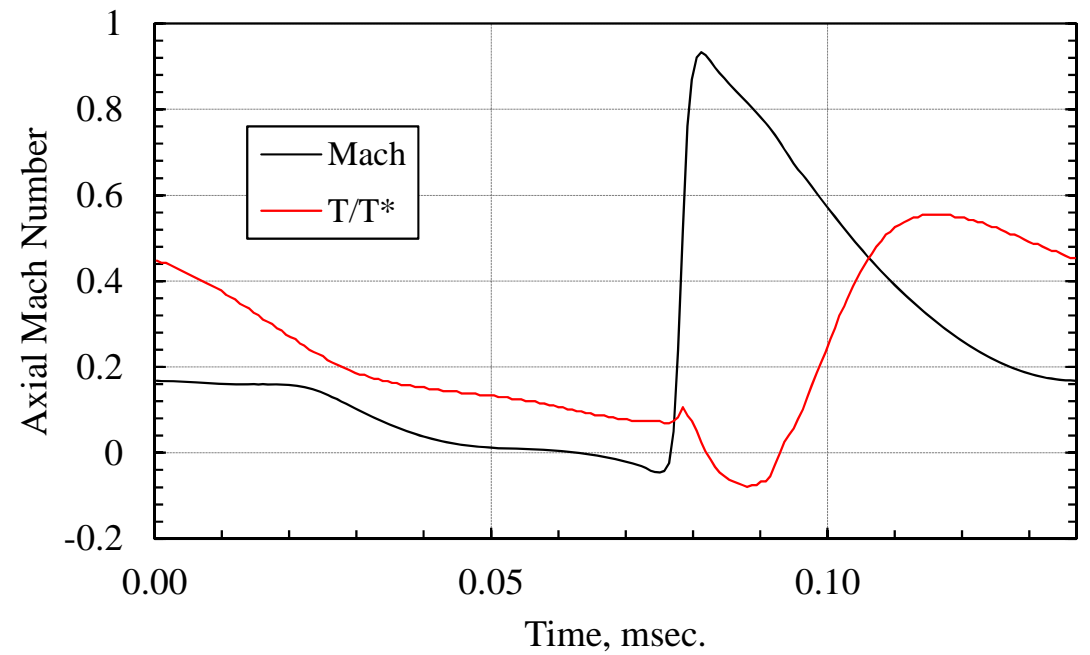

6.10

Figure 7. Computed exit plane axial Mach number and temperature of the experimental RDE, at a fixed point, in the laboratory frame of reference. 
eventually turn back around and re-enter the RDE. As such, the backflow and re-entry regions can be thought of as a source of blockage, requiring a higher inlet pressure to achieve a given mass flow rate through the device. Suffice it to say that any inlet designs which provide more resistance to backflow, without causing additional total pressure drop in forward flow would be of large benefit to RDE technology.

The above being said, $18 \%$ backflow is not unreasonably high for these inlets. In fact it may be less than optimal when inflow total pressure losses across the restriction are considered. A mass flux average of the entropy (relative to the inlet manifold) associated with unreacted flow just downstream of the inlet indicates a $43 \%$ loss in total pressure for the flow. Considering the estimated overall RDE pressure ratio of 0.83 , this implies that the detonation itself is developing a pressure ratio of 1.46; a true pressure gain. Unfortunately, this is not enough to overcome the massive inlet losses. The finding also implies that there may be an optimal inlet area restriction that better balances the backflow and throughflow losses just described.

\section{B. Optimization}

The RDE simulation was reconfigured such that the length was reduced by $67 \%$, and the inlet area was increased by $49 \%$. These values were obtained after trying several other length and inlet area changes; however, they do not represent a true optimization in the sense of finding values which yield the best performance. The goal was merely to illustrate that improvements were possible, based on the simulation results. The backpressure was held to the same value as that of the original $90 \%$ speed simulation. The inlet manifold pressure was then adjusted until the original mass flow rate was achieved. The required manifold pressure was found to be 73.8 psia. The resulting cycle is shown in Fig. 10 as temperature contours similar to Fig. 6.

It was found that shortening the RDE eliminated all backflow in the exit plane, and reduced heat lost to the walls to just $14 \%$ of the chemical energy. Increasing the inlet area increased the backflow percentage to $25 \%$, but reduces the inlet total pressure loss to $26 \%$. The net result is an RDE component pressure ratio of 1.11 , making it a true pressure gain device.

Applying the mixing calculation yields no change in the mixed total pressure at the turbine inlet compared to the original $90 \%$ speed analysis. The reason for this appears to be twofold. First, the average velocity exiting the RDE is higher, resulting in a higher component pressure ratio. In a mixing calculation however, the larger the gradient

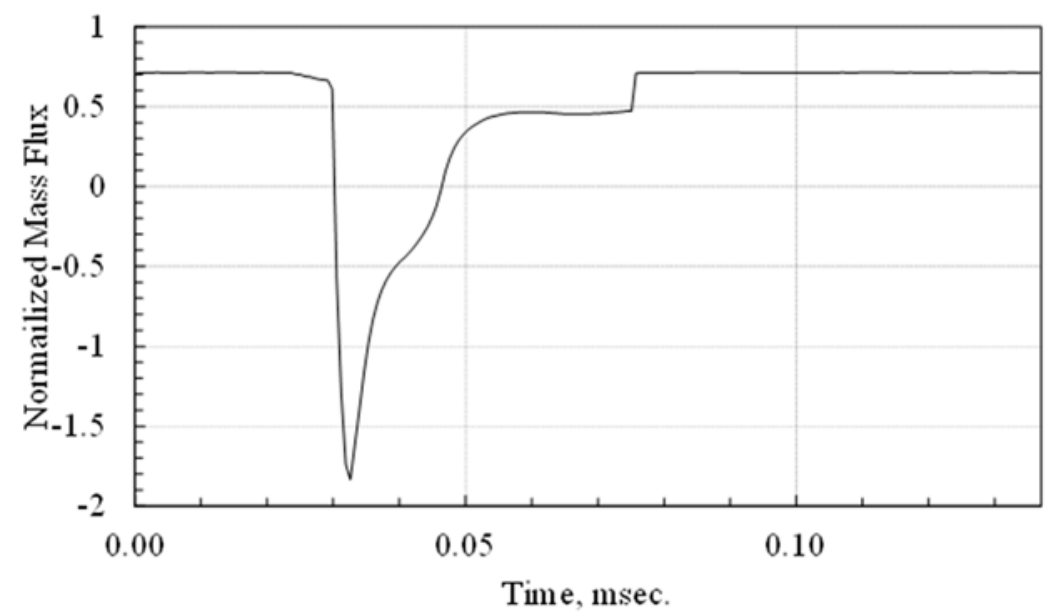

Figure 9. Computed inlet plane non-dimensional axial mass flux rate at a fixed point in the laboratory reference frame. between RDE and ejector velocities, the more entropy is produced in making the flows uniform. Second, the hotter RDE flow (from reduced heat transfer) necessitates a cooler ejector flow in order to achieve the same mixed total pressure. As such, the ejector velocity is reduced, which exacerbates the entropy production just described.

That being said, it is also noted that the total pressure required to drive the ejector flow (supplied by gas bottles) is reduced, and so is the inlet pressure of the RDE. Both of these pressures are currently above the compressor 


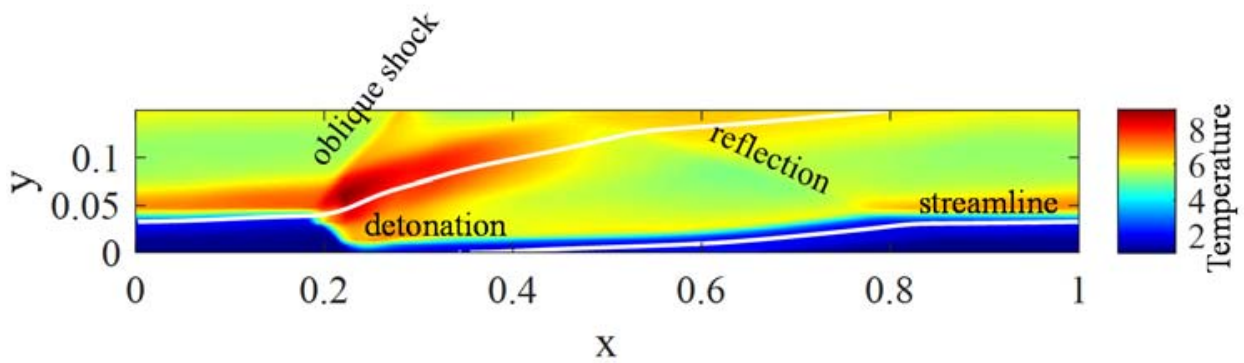

Figure 10. Computed contours of normalized temperature throughout the annulus of the optimized RDE, in the detonation frame of reference.

discharge pressure. Thus, the improved RDE performance could move a redesigned experiment closer to the possibility of closed loop operation.

\section{Conclusion}

Results from an experimental rig consisting of a rotating detonation engine (RDE) with bypass ejector flow coupled to a downstream turbine were analyzed using a validated computational fluid dynamics RDE simulation combined with an algebraic mixing model of the ejector. The analysis agreed reasonably well with limited available data, suggesting that the simulation had correctly captured the flow field physics and could be further examined in order to understand the operation of the RDE. The examination indicated that the RDE operated in an unusual fashion, with subsonic flow throughout the exhaust plane. The rotating detonation produced a total pressure rise relative to the pre-detonative pressure; however, the length of the device and the substantial flow restriction at the inlet yielded an overall pressure loss. This was expected given that achieving pressure gain was not an objective of the experiment. It was shown however, that with changes to the RDE length and inlet area the RDE could produce an overall pressure rise. The analysis also indicated that the mixing model, which yields a uniform flow from a plane into which nonuniform flow is directed, adds appropriate entropy (i.e. total pressure loss) so as to mimic the lost work extraction capability of the turbine operating in the unsteady environment that actually exists behind an RDE.

\section{Acknowledgements}

The authors would like to thank Scott Jones, of NASA's Glenn Research Center for supplying the NPSS information used in this work.

\section{References}

${ }^{1}$ Paxson, D. E., Kaemming, T., "Foundational Performance Analyses of Pressure Gain Combustion Thermodynamic Benefits for Gas Turbines,” AIAA 2012-0770, Jan., 2012.

${ }^{2}$ Welsh, D. J., et. al., "RDE Integration with T63 Turboshaft Engine Components,” AIAA 2014-1316, Jan. 2014.

${ }^{3}$ Naples, A., et. al., "Design and Testing of a Rotating Detonation Engine for Open-Loop Gas Turbine Integration,” presented at $25^{\text {th }}$ International Colloquium on the Dynamics of Explosions and Reactive Systems, Aug. 2015.

${ }^{4}$ Naples, A., Hoke, J., Schauer, F., “Rotating Detonation Engine Interaction with an Annular Ejector,” AIAA 2014-0287, Jan. 2014.

${ }^{5}$ Naples, A., Hoke, J., Battelle, R. T., Wagner, M., Schauer, F., “RDE Implementation into an Open-Loop T63 Gas Turbine Engine,” to be presented at the AIAA SciTech 2017 Forum, paper number not yet assigned, Jan. 2017.

${ }^{6}$ Paxson, D.E., "Numerical Analysis of a Rotating Detonation Engine in the Relative Reference Frame," AIAA-2014-0284, Jan., 2014, also NASA/TM 2014-216634, 2014.

${ }^{7}$ Paxson, D.E., Fotia, M.L., Hoke, J.L., Schauer, F.R., “Comparison of Numerically Simulated and Experimentally Measured Performance of a Rotating Detonation Engine,” AIAA-2015-1101, Jan. 2015.

${ }^{8}$ Theuerkauf, S.W., et al, “Comparison of Simulated and Measured Instantaneous Heat Flux in a Rotating Detonation Engine,” AIAA 2016-1200, Jan., 2016.

${ }^{9}$ Rankin, B., Fotia, M. L., Paxson, D. E., Hoke, J. L., Schauer, F. R., “Experimental and Numerical Evaluation of Pressure Gain Combustion in a Rotating Detonation Engine,” AIAA 2015-0877, Jan., 2015.

${ }^{10}$ Perkins, H.D., et. al., “An Assessment of Pulse Detonation Engine Performance Estimation Methods Based On Experimental Results,” AIAA 2005-3831, Jul., 2005.

${ }^{11}$ Paxson, D.E., Naples, A.G., Hoke, J.L., Schauer, F. "Numerical Analysis of a Pulse Detonation Cross Flow Heat Load Experiment,” AIAA-2011-584, Jan., 2011.

${ }^{12}$ Paxson, D.E., Schauer, F.R., Hopper, D., "Performance Impact of Deflagration to Detonation Transition Enhancing Obstacles,” AIAA-2009-0502, Jul., 2009, also, NASA/TM-2012-217629.

12

American Institute of Aeronautics and Astronautics 
${ }^{13}$ Paxson, D.E., “A General Numerical Model for Wave Rotor Analysis,”NASA TM 105740, 1992.

${ }^{14}$ Paxson, D.E., “An Improved Numerical Model for Wave Rotor Design and Analysis,” AIAA-93-0482, Jan., 1993.

${ }^{15}$ Gordon, S., McBride, B. J., "Computer Program for Calculation of Complex Chemical Equilibrium Compositions and Applications,” NASA RP 1311, Oct. 1994.

${ }^{16}$ White, F., Fluid Mechanics, McGraw-Hill, 1979, pp. 376-386.

${ }^{17}$ Paxson, D.E., Hoke, J.L., "Time Averaged Pressure Measurement in Fundamentally Unsteady Pressure Gain Combustion Systems,” in Proc. JANNAF 45th Combustion Subcommittee, 33rd Airbreathing Propulsion Subcommittee, 27th Propulsion Systems Hazards Subcommittee, Monterey, CA, December, 2012, also NASA/TM—2013-217826, Jan., 2013.

${ }^{18}$ Evans, A.L. et. al., “Numerical Propulsion System Simulation's National Cycle Program,” AIAA 98-3113, Jul., 1998.

${ }^{19}$ Paxson, D.E., "Performance Evaluation Method for Ideal Airbreathing Pulse Detonation Engines,” Journal of Propulsion and Power, Vol. 20, No. 5, pp. 945-950, 2004. 